\title{
Development of a multi frequency impedance measurement system for use in MEMS flow cytometers
}

\author{
Neil J. Cole ${ }^{1} \cdot$ Andrew M. Richardson $^{1}\left(\right.$ Alako Abdul-Hafiz $^{1}$
}

Received: 9 January 2017 / Accepted: 7 March 2017 / Published online: 17 April 2017

(C) The Author(s) 2017. This article is an open access publication

\begin{abstract}
A novel MEMS based system for flow cytometry is presented based on a multispectral approach. The solution provides the ability to concurrently generate, detect and analyse the response from a biological cell using multiple narrow band signals with a frequency separation sufficient to evoke different cellular responses. The narrow band approach limits the energy required and provides an improved signal to noise ratio for a given transmitter energy, avoiding wide band signal power dissipation and dilution issues. It requires only a single transmitter and receiver interface to the MEMS device with no duplication of analogue signal paths. The design has the ability to generate a complex multi frequency waveform with a wide frequency separation. A digital approach based on the CORDIC algorithm (Volder, IRE Trans Electron Comput EC-8:330-334, 1959) was used to generate the required sinusoidal waveforms. The digital section of the design was implemented on an FPGA. It utilised a modified digital phase sensitive amplifier to provide enhanced signal to noise performance and harmonic rejection. A novel hybrid, cross channel electrode structure to implement the stimulus and response analysis sub-system has been designed. A fully functional macroscopic test bench has been constructed to demonstrate the viability of the approach. Both physical measurements and finite element data has been used to demonstrate the potential for migration into a MEMS based system.
\end{abstract}

Andrew M. Richardson

a.richardson@lancaster.ac.uk

1 Department of Engineering, Lancaster University, Lancaster, UK

\section{Introduction}

The complex impedances that a biological cell represents change with frequency (Beving 1994). The cellular dispersion responses were originally classified as $\alpha, \beta$ and $\gamma$, dependent on the dominant dispersion mechanism. The low frequency $\alpha$ response is linked to the cell membrane impedance. This response typically dominates from DC to approximately $200 \mathrm{kHz}$ (Wolf 2011; Britt and Hoffman 1979). There are indications the $\alpha$ response is absent in, for example, undamaged red blood cells and only present in haemolysed material (Wolf 2011). The more complex $\beta$ response is linked to Maxwell-Wagner (Fricke 1953) dispersion. This has been sub divided into two regions $\beta 1$ and $\beta 2$, based on the different mechanisms dominant at the separate peaks. The first occurs in the $1 \mathrm{MHz}$ region (Wolf 2011; Beving 1994). The transition to a $\gamma$ type response is typically considered to occur in the region of $1 \mathrm{GHz}$ and is linked to water molecule relaxation effects. An additional $\delta$ response in the region between the $\beta$ and $\gamma$ response is also present (Wolf 2011). At frequencies in the $\mathrm{MHz}$ region ( $\beta$ response) it is possible to investigate the structure of the cytoplasm within the cell. At lower frequencies, the cell membrane causes cells to appear as isolated capacitors. Utilising the impedance response across a wide bandwidth provides discrimination between cell types and improved sensitivity of the measurement extending the range of diagnostic information that can be aquired. This work aims to provide a methodology able to optimise systems based on non-contact multi-frequency measurements that has been shown in the literature to be capable of differentiating between cells of the same or a similar type with normal, cancerous, or diseased conditions.

The counting of individual cells using DC impedance techniques has been in use since it was first described in a paper by Coulter in the 1950's (Coulter 1956). In its most 
basic form, a Coulter based counter consists of two electrodes, in a narrow channel connecting separate containers. One container holds an electrolyte solution with the cells to be analysed, the other is a waste container. A current is maintained between the two electrodes through the electrolyte and the connecting channel and a positive fluid flow is generated from the reservoir container via the channel. When a cell transits the channel, it represents a discontinuity, causing the impedance between the electrodes to change. This technique relies on the relationship between the narrow channel and the cell size, for its sensitivity. The change in impedance having a direct relationship to the cells volume, sometimes called the "Coulter Volume". The technique does have limits regarding the detection levels in terms of the percentage volume change, caused by a cell. Values in the order of $0.0004 \%$ have been reported (Emanminejad et al. 2012). Such techniques readily lend themselves to the miniaturisation inherent in MEMS devices as the reduction in channel volume increases sensitivity. The lower limit for the reliable detection of pulses caused by the transit of a cell is however influenced by contamination, external and circuit related noise sources, as well as the impact of electrolysis due to the inter electrode current flow (Chen et al. 2014). There are also issues due to the narrowing of the channel that has detrimental effects in terms of changes in pressure and flow. Blockage and cell damage can occur at the constriction (Nieuwstadt 2011). The ability to discern individual cells and provide a reliable count is directly related to the flow rate, relative cell density, length and diameter of the channel. The achievable count rates are relatively low, requiring considerable dilution of materials such as human blood, which can contain more than 5 million cells per cubic millimetre, to ensure a reliable count. Discrimination between individual cells is limited by the transit time of cells through the narrow channel detection region. This transit time can be regarded as "Dead Time" for the measurement system and places an upper limit on the count rate.

AC approaches extend the basic Coulter method (Johnson et al. 1980; Britt and Hoffman 1979) by introducing an AC component to the electric field used. AC detection utilises the complex impedance that the cell represents. The ability to "look" inside the cells allows discrimination and sensitivity to be improved. Differentiation between living, dead, viable, non-viable, normal and cancerous cells have all been reported.

$\mathrm{AC}$ approaches are, limited due to electrolysis between the electrodes and the liquid carrier and the formation of a capacitive electric double layer (EDL) due to polarisation limits the low frequency response. In the case of MEMS solutions utlilising electrodes immersed in the fluidic stream, reliability, yield and manufacturing complexity can all be compromised. Contactless approaches have to take into account a significant reduction in the effective field strength. To compensate for this loss in sensitivity, modern technologies allow the complex impedances that cellular responses represent, to be measured over higher frequency ranges. The integration of techniques used in modern RF and cellular design, improves sensitivity and discrimination. Approaches using techniques such as differential phase (Britt and Hoffman 1979), RF resonance (Wood 2005), lock in amplifiers (Javanmard et al. 2012), DSP (Holmes et al. 2007), direct measurement of the complex impedance (Spencer et al. 2012), pulse shaping, FFT analysis (Van Berkel et al. 2009) and microwave evanescent fields have all been successfully applied.

The use of wide band or spread spectrum based techniques such as maximum length sequences, (Sun et al. 2007) (MLS) pseudo random binary sequences (PRBS) and code division multiple access (CDMA) approaches coupled with correlation or Fourier based post processing have been explored. Such approaches typically spread the transmitter energy over the whole spectrum of interest. Impulse based techniques using Chirps, Square waves or Walsh codes improve the energy present in specific frequencies when compared to these wide band approaches (Min et al. 2009). When processing the received signal, correlation based approaches provide only a single output in terms of the correlation result. Whilst this is resilient to noise and provides enhanced sensitivity it provides no data on the response of a cell at multiple frequencies. Fourier analysis can address this by providing the ability to acquire information from multiple frequency bands concurrently. However, the signal energy is widely distributed and the bandwidth and resolution of such approaches are limited by the time, memory and processing power available. This is particularly important in situations where transient events are monitored and real time processing is required. For measurement of cellular characteristics, values are stable for relatively long periods hence such approaches will utilise considerable resource to generate little or no additional information. The approach presented in this paper is both resource efficient and able to generate cellular responses over multiple narrow band signals, with a significant frequency seperation.

\section{Implementation}

\subsection{Generating sinusoidal waveforms}

The approach for generating the sinusoidal waveforms uses a hardware description language (HDL) implementation of the CORDIC algorithm developed by Jack Volder in 1959 (Volder 1959). The CORDIC algorithm was intended as a replacement for an analogue resolver in use by the US Air Force at the time. Its approach makes it, as it was intended to be, suitable for implementation using limited computational resources. It has been used in a number of approaches 
requiring relatively low cost, synthesisable implementation of a high resolution sinusoid waveform generator (Ristovic 2012; Upadhyay 2013; Andraka 1998; Hu 1992). Specifically the approach reduces the need for multipliers to be used, limiting the range of the required mathematical transforms for the iterative loop to \pm 2 , that allows them to be replaced with binary shift operations. This means it can be synthesised with a relatively low overhead. A CORDIC algorithm using an iterative structure reaches an optimum solution in $N-1$ clock cycles, where $N$ is the bit width of the data word used by the algorithm. The error between a double precision floating point approach and the CORDIC algorithm for a range of data widths is shown in Table 1. This shows the worst error over a range of $0-2 \pi$ radians. The important facet of a CORDIC, opposed to a direct digital synthesizer (DDS) or numerically controlled oscillator (NCO) based approach using a lookup table (LUT) is that it will reach a full precision answer for any input value in $N-1$ clock cycles.

The limitation is that it is an iterative process, although an alternative architectural implementation utilises asynchronous combinational logic that reduces the number of cycles required to reach the final value. This is at the expense of area and recoding the register-transfer level (RTL) structure into a far less flexible form. Whilst ideal for the requirements in terms of clock resolution and data rate for the higher bit ranges (e.g. 16 and 24 bit), implementation is restrictive given a data to clock ratio of 1:16 or 1:24 is required. This specifies a clock rate of 320 or $480 \mathrm{MHz}$ to support a $10 \mathrm{MHz}$ sinusoid at the Nyquist limit. An alternative approach was hence required. The relatively low synthesised area of this approach allows multiple blocks to be instantiated on even a modest sized FPGA. A synchronisation scheme was therefore developed that allowed a high rate input data stream to be multiplexed between CORDIC blocks and then de-multiplexed to provide a single high rate output data stream. This meant that a CORDIC assembly could supply data samples at a rate varying from $N-1$ to 1 relative to the system clock. The latter required the assembly to contain $N-1$ CORDIC blocks. It should be noted that this is in effect a pipeline. The number of clock cycles between any one input data sample and its corresponding output data sample remains at $N-1$. The longest combinational delay in the structure places a limit on the maximum sustainable clock rate. For the design as implemented on an Altera Cyclone II FPGA, the longest delay chain, as reported by TimingQuest, was $14 \mathrm{~ns}$. This results in a theoretical maximum clock rate of approximately $70 \mathrm{MHz}$, in excess of the FPGA development boards $50 \mathrm{MHz}$ system clock limit. A variation on this approach allows a single CORDIC assembly to concurrently support multiple sinusoids by multiplexing the data streams from the lower rate systems into a single higher rate data stream. The high rate output stream is then de-multiplexed into the original low rate streams. Reducing the overall number of CORDIC assemblies required in the design, at the expense of an increased clock rate.

\subsection{Phase sensitive detection}

Phase sensitive, or lock in amplifiers provide an approach to resolve a signal of known frequency, or characteristic, from an environment where significant broad band noise is present. Its use is not limited to sinusoids; the approach can amongst other things be used for square waves or pseudo random binary bit streams. The approach allows for significant attenuation of any broad band noise in the signal. It requires that the input and reference signal be identical in frequency. Figure 1 shows the basic structure of a phase sensitive amplifier.

The theory of operation is straight forward, for a pure sinusoidal approach. If we express the input signal as:

$V_{\text {Signal }}=V_{I N} \sin \left(\omega_{I N} t+\phi_{I N}\right)$

where $\mathrm{V}_{\text {IN }}$ is the magnitude of the input waveform. If we multiply this by a reference signal then we get the expression:

$V_{\text {Signal }}=\left[V_{I N} \sin \left(\omega_{I N} t+\phi_{I N}\right)\right] \times V_{R E F} \sin \left(\omega_{R E F} t+\phi_{R E F}\right)$

Expanding this gives us the sum and difference terms.

$$
\begin{aligned}
V_{S U M} & =\frac{1}{2} V_{I N} V_{R E F}\left(\cos \left(\omega_{I N}+\omega_{R E F}\right) t+\left(\phi_{I N}+\phi_{R E F}\right)\right) \\
V_{D I F F} & =\frac{1}{2} V_{I N} V_{R E F}\left(\cos \left(\omega_{I N}-\omega_{R E F}\right) t+\left(\phi_{I N}-\phi_{R E F}\right)\right)
\end{aligned}
$$

If we apply a low pass filter to these expressions, for the case where $\omega_{\mathrm{IN}}=\omega_{\mathrm{REF}}$ then they simplify to a DC term:

$V_{\text {OUT }}=\frac{1}{2} V_{I N} V_{R E F} \cos \left(\phi_{I N}-\phi_{R E F}\right)$

The phase relationship between the signals is undefined, and can vary dependent on the phase shift in the signal chain. For a system using multiple frequencies, the phase shifts will be different for each frequency. There are two potential approaches to compensate for this unknown phase delay and maximise the magnitude of the recovered signal. The first approach involves
Table 1 Error between CORDIC and floating point sinusoid

\begin{tabular}{lllllll}
\hline Word & 8 & 10 & 12 & 14 & 16 & 24 \\
\hline$\%$ error & 6.5399 & 1.549 & 0.5029 & 0.1638 & 0.0387 & $2 \mathrm{e}^{-5}$ \\
\hline
\end{tabular}


Fig. 1 Basic phase sensitive amplifier

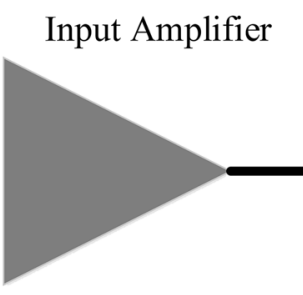

Phase Sensitive Detector

Low Pass Filter sychronising the frequency and phase of the reference signal with the input signal and the second, the use of orthogonal signals and duplication of the process using the cosine of the reference signal. In this case we are left with expressions for the magnitude of the orthogonal components of the signal which can be expressed as:

$V_{C M A G}=\frac{1}{2} V_{I N}(t) V_{R E F} \cos \left(\phi_{I N}-\phi_{R E F}\right)$

$V_{S M A G}=\frac{1}{2} V_{I N}(t) V_{R E F} \sin \left(\phi_{I N}-\phi_{R E F}\right)$

Figure 2 shows the output from a digital phase sensitive detector as the input frequency is swept across the reference frequency. The frequency change in the difference component can be seen clearly.

The magnitude and phase of the signal can be generated from the relationships:

$$
\begin{aligned}
V_{M A G} & =\sqrt{\sin ^{2}+\cos ^{2}} \\
\text { Phase } & =\tan ^{-1}\left(\frac{\sin }{\cos }\right)
\end{aligned}
$$

Fig. 2 Digital phase sensitive detector
Due to the timing delays in the signal chain and the potential complexity in synchronising multiple signals it was decided to use an orthogonal approach. In a sampled system, the situation is not quite so straight forward (Neeman 2004). The phase accuracy achievable for orthogonal systems will be limited by the quantisation of the phase angles. Limiting the phase matching to $2 \pi / N$, where $N$ is the number of data points for the frequency of interest will create a potential magnitude mismatch related to $N$. This can have the worst impact at low sample rates where the quantised phase shift will be greatest. In these situations the use of random or pseudo random sampling approaches has significant advantages. In the case of the orthogonal approach used, where the channel offset is $\pi / 2$, the potential mismatch between the measured and real magnitude is approximately $15 \%$ at 10 data points and drops to $2.5 \%$ at 20 data points. This is the result for the worst possible phase mismatch affecting both orthogonal channels.

\subsection{Low pass filter}

The broad band noise contribution is bandwidth limited by the low pass filter. The narrower the pass band the lower the

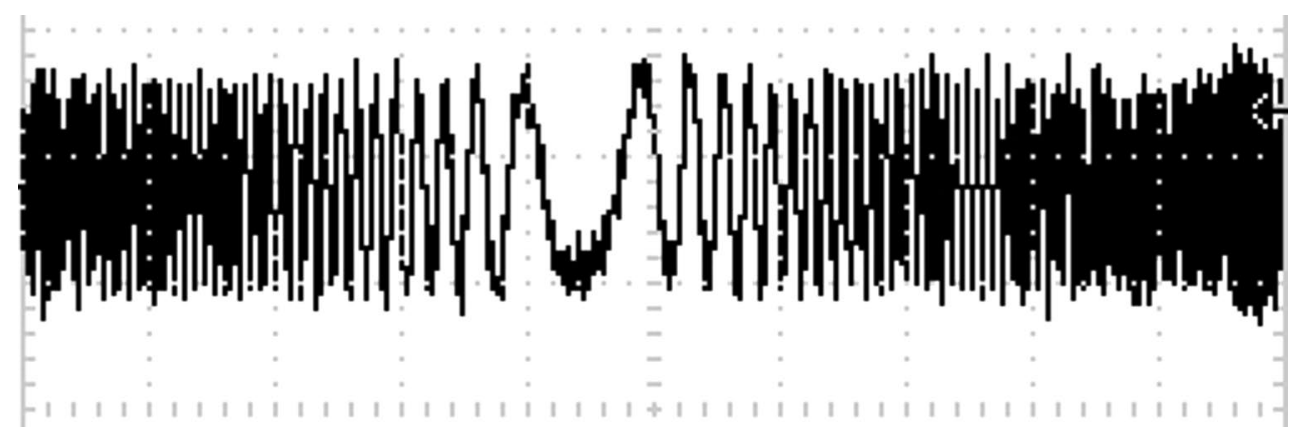


broad band noise contribution. For static or slowly changing systems the low pass filter can be in the sub hertz region, making this contribution minimal. In this case transient events need to be detected to support analysis of the cell's characteristics. This means that the low pass filter will need to be in the $1-50 \mathrm{kHz}$ range, depending on the planned count rate. This increases the noise contribution on the channel significantly, as any noise with a frequency component below the filter cut off will be present in the final signal chain. For a digital design, the filter can be implemented using a DSP approach. The use of fixed point rather than floating point arithmetic can cause serious issues within a DSP system. Care needs to be taken to review the coefficients generated for any filter used, to ensure that sufficient resolution is provided by the selected fixed point range. This is a particular problem when significant differences in sample rates exist or high order filters are used. The ratio between the coefficients can become small leading to issues if insufficient fixed point resolution is available. In some cases the preferred approach is to use multiple low order filters to achieve a high order final result.

\subsection{Digital phase sensitive approaches}

Traditionally digital phase sensitive detectors have used a square wave synchronized with zero crossing detectors based on a recovered or reference signal. These are sensitive to the reference signal and its harmonics. Approaches using an analogue or digital PLL have been used to provide pseudo sine waves for low frequency systems, improving harmonic rejection. These are feasible for single frequency systems. For a higher frequency system using multiple frequencies the solution would be complex and potentially unstable. In a sampled system there is no virtue in providing reference signal data points at a rate greater than the number of data points present in the input sample for the waveform. For a system responding to transient events, oversampling should be used when feasible. However, as frequencies increase the number of data points per cycle that the A2D converter can supply reduces. As can be seen in Fig. 3, increasing the level of quantisation for the reference signal from a square wave provides a significant improvement in the discrimination of a transient event, for even a minimal increase.

Thus we need to ensure that the number of points generated for the reference signal match those of the input signal for each of the frequencies of interest, ensuring that the optimal number of points are used for each reference frequency. A block diagram of the overall system for a single channel of an orthogonal phase sensitive amplifier is shown in Fig. 4. The model was developed using Matlab Simulink. The development model used is shown in Fig. 5. Matlab Simulink includes functions to quantise waveforms and generate CORDIC sinusoids. These CORDIC commands are not synthesizable and a separate model needed to be developed to implement the CORDIC algorithms, using fixed point models, which could be simulated and used to generate RTL code for synthesis.

This allowed evaluation of quantisation issues, filter characteristic, mathematical calculations and appropriate fixed point resolutions in a coordinated approach. Once finalised the required blocks were synthesised and
Fig. 3 Sensitivity with 1, 2, 3, 4 bit and sine wave




Fig. 4 Phase sensitive amplifier block diagram

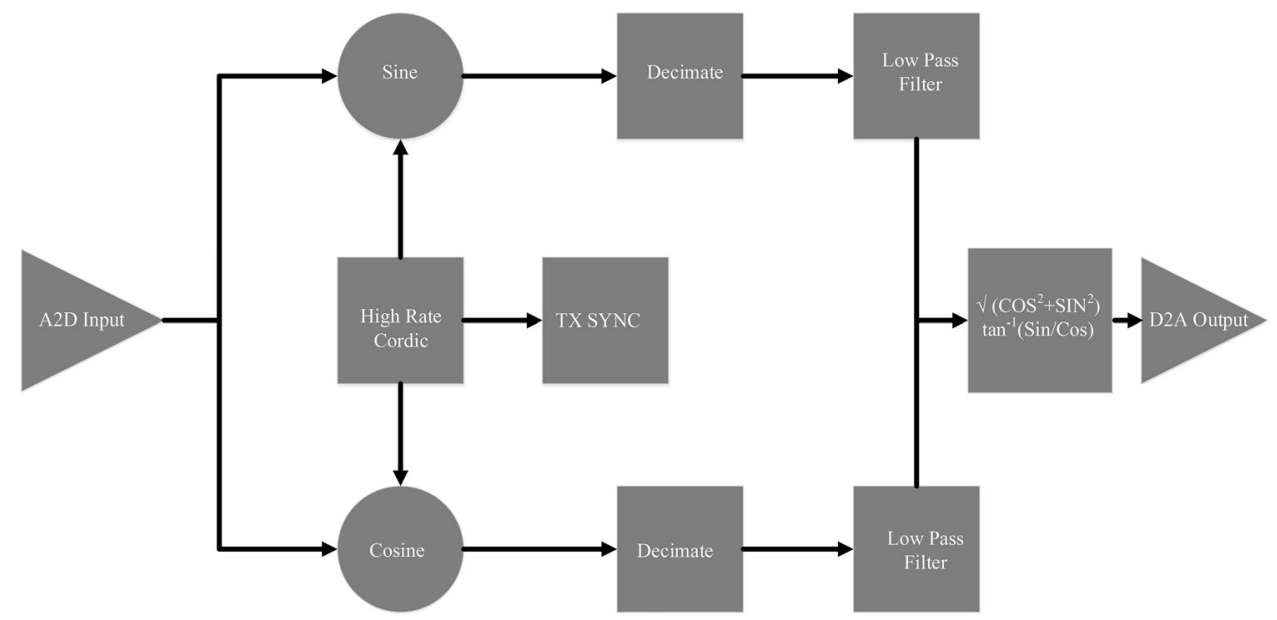

DC Offset for Input Sample Rate and Quantisation to match A2D

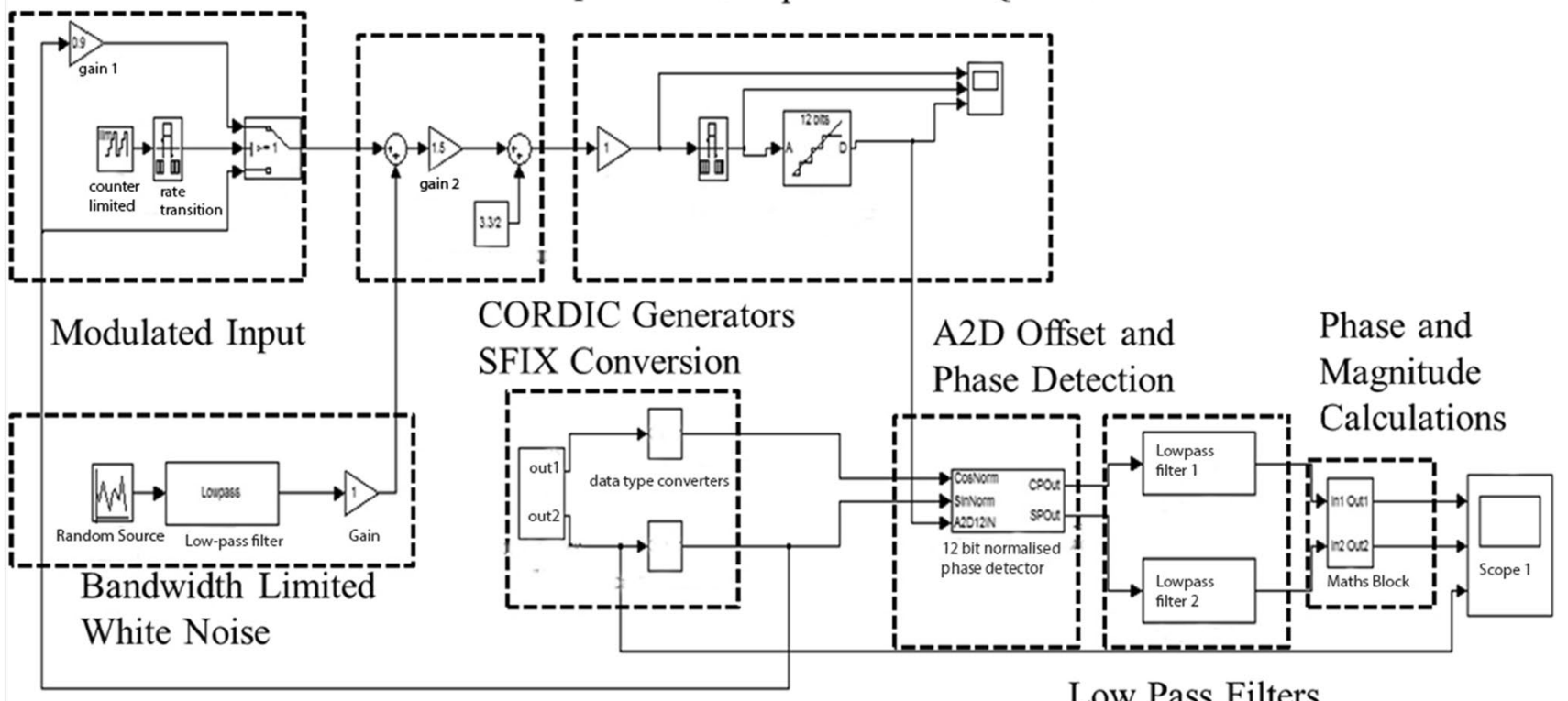

Low Pass Filters

Fig. 5 Matlab simulink model

integrated with the VHDL structure. The final structure of the VHDL, with a single orthogonal phase sensitive amplifier channel instantiated is shown in Fig. 6.

The design was implemented on an Altera DE1 development board. Standard Analog Devices components were used for the A2D and D2A systems. The use of a high sample rate $\mathrm{A} 2 \mathrm{D}$ converter for the front end meant there was a significant mismatch between the filter rate and the lower sample rate of the phase and magnitude D2A devices. In order to avoid issues with the combinational delays and fixed point resolution of the low pass filters, the input data streams were decimated after the phase sensitive detectors to match the phase and magnitude D2A conversion rate. The higher the input rates for a filter the tighter the timing requirements, and the larger the fixed point range required to ensure correct operation. A decimator contains the majority of the structures required to implement a FIR filter, and as is common practice the filter and the decimator were combined for the final design. As implemented, the design could support up to four separate frequencies from DC up to the Nyquist limit of the high speed D2A converter. In all cases, the A2D conversion path was the limiting factor on the maximum frequency achievable. The system supported two data rates from the CORDIC elements. The first matched the D2A converter rate, allowing a 




Fig. 6 VHDL block structure

Fig. 7 Unsmoothed frequency combinations

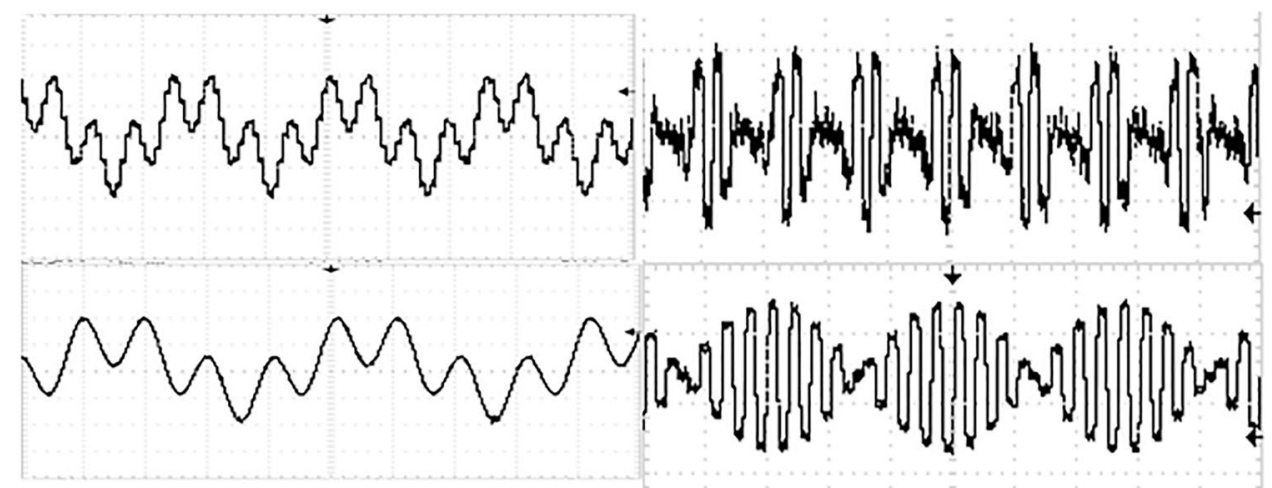

minimisation in the residual noise after the smoothing filter. The second matched the selected frequency to the A2D sample rate providing optimised data points for the sine and cosine signal paths. Figure 7 shows a number of unfiltered output waveforms for a 2 channel combined sinusoid with differing frequency ratios and sample rates.

Figure 8 shows the output from the working system with the MEMS channel modeled using discrete components. The transient amplitude change has clearly been detected from the broadband noise present in the signal chain.

\section{Development of the MEMS environment}

\subsection{Cell AC characteristics and FEM modelling}

As discussed in Sect. 1, biological cells are inhomogeneous in structure. This gives rise to a nonlinear frequency dependent response when an AC field is applied. A simplistic representation that ignores the malleable nature of cells allows them to be represented as spheres comprising of a number of layers. Polystyrene test spheres present a single homogenous structure. Red blood cells lenticular 


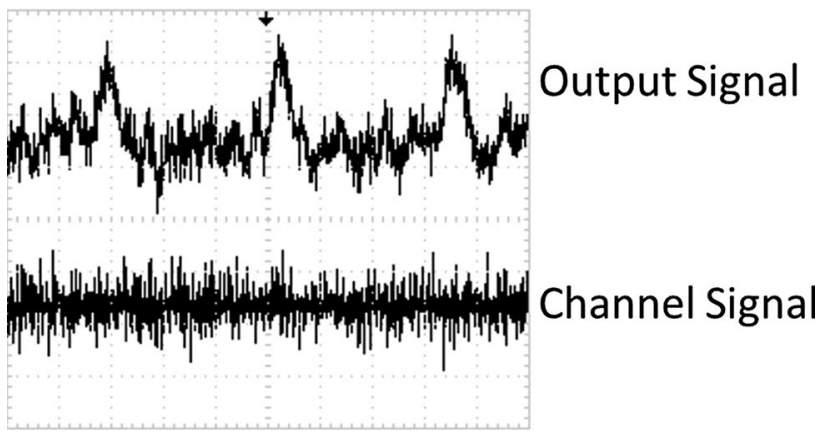

Fig. 8 Signal detection results from the final system

nature comprise of two elements, a cell membrane surrounding the cells cytoplasm. Bacteria such as E. coli comprise of three elements; the cell wall, cell membrane and the cytoplasm. Electrically the cell membrane can be considered to be a thin insulating shell around the intracellular cytoplasm, which has a significantly lower electrical resistance and a different permittivity. A significant number of studies have been carried out, using dielectrophoresis (DP) and electrorotation (ER) techniques allowing cell identification, manipulation and selection using AC electric fields. A number of reports detail variations in the measured values of electrical conductivity and permittivity for red and white blood cells as haematocrit or phosphorous buffered saline (PBS) concentrations vary (Britt and Hoffman 1979; Park et al. 2011; Gimsa
1998; Ulgen and Sezdi 1998). These structures can be replicated directly in physical models or represented as electrical circuits, allowing the modelling of cellular response in FEM, spice or AHDL based tools. Simple electrical equivalents to these structures are shown in Fig. 9. These models can provide simulations of the $\alpha$ and $\beta$ transitions.

\subsection{Cell modelling}

A substantial amount of work has been done extending the applicability and accuracy of equations used to predict the behaviour of cells in AC fields. Extending from the original dielectric sphere calculations and Laplace based approaches through to inclusion of work by Debye (1945), Maxwell and Wagner (Fricke 1953) onto more modern and complex emulations. The advent of finite element analysis simulations allowed modelling of the interactions of cells and their environments in 2 or 3-dimensions.

\section{$3.3 \alpha, \beta$ and $\gamma$ frequency responses of cells}

In terms of cellular responses, later analysis has tended to move away from the $\alpha, \beta$ and $\gamma$ classification and to consider the main processes involved instead (Gimsa 1998). In particular, the Maxwell-Wagner (Fricke 1953) processes for the $\beta$ range and (Debye 1945) dispersions for the $\gamma$ range. This approach has gained favour as it improves

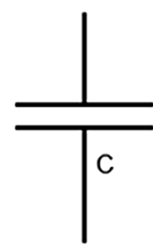

\section{Homogenous polystyrene bead}
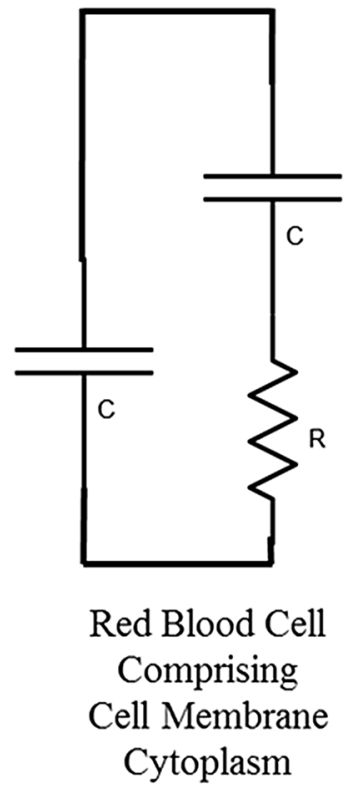

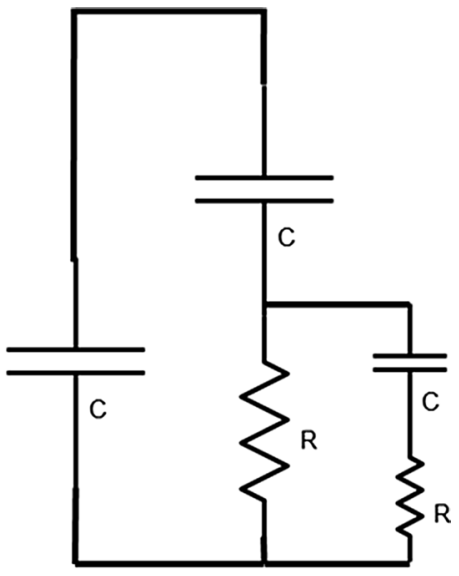

Bacterium

Comprising

Cell Wall

Cell Membrane

Cytoplasm

Fig. 9 Electrical equivalent circuits of bead, cell and bacterium 
analysis for DP and ER cellular characteristics. The ColeCole (Cole 1941) equation is considered a version of the original Debeye relaxation approach. Complex bio-impedances are routinely expressed using a Cole-Cole plot allowing for the direct evaluation of a number of parameters such as effective capacitance, characteristic frequency and cell based impedance effects from experimental data.

\subsection{Finite element analysis of MEM devices}

A model of a MEMS device including cells, channel, electrodes, carrier medium and carrier PCB was created in COMSOL. This allowed qualitative comparison of their characteristics and the impact of variations in channel width, height, bead, cell placement and location relative to the electrodes and electrode placement. The two dimensional test structure used is shown in Fig. 10.

It shows the initial dimensions and structure as well as the high resolution and low resolution mesh zones used in the channel to reduce the number of elements in the model. Finite element models were developed to match the cellular characteristics defined in a number of papers (Park et al. 2011; Gimsa 1998; Ulgen and Sezdi 1998). The values used for the cell and bacterium were taken from Table S1 from Park and Zhangs paper (Park et al. 2011). The results from the finite element analyses showed the requisite characteristics within what was considered an acceptable range of accuracy, given the unsophisticated nature of the models. Figure 11 shows the field lines for a simulation of the bacterium cell at frequencies of $10 \mathrm{kHz}(\alpha), 520 \mathrm{kHz}(\beta 1)$ and $3.2 \mathrm{MHz}(\gamma)$. The transition between external and internal conductance within the cell as the frequency increases is clearly shown by the penetration of the field lines into the cytoplasm.

\subsection{Blood cell characteristics}

The polystyrene test beads used were homogenous and exhibited a single characteristic across the frequency range. The ability to compare the results from these and correlate them with simulations allowing analysis of the responses when the test beads were replaced with blood cell and bacterium models.

The expected characteristics were seen in the simulations, in particular:

- The differences between the bead and the cell frequency responses.

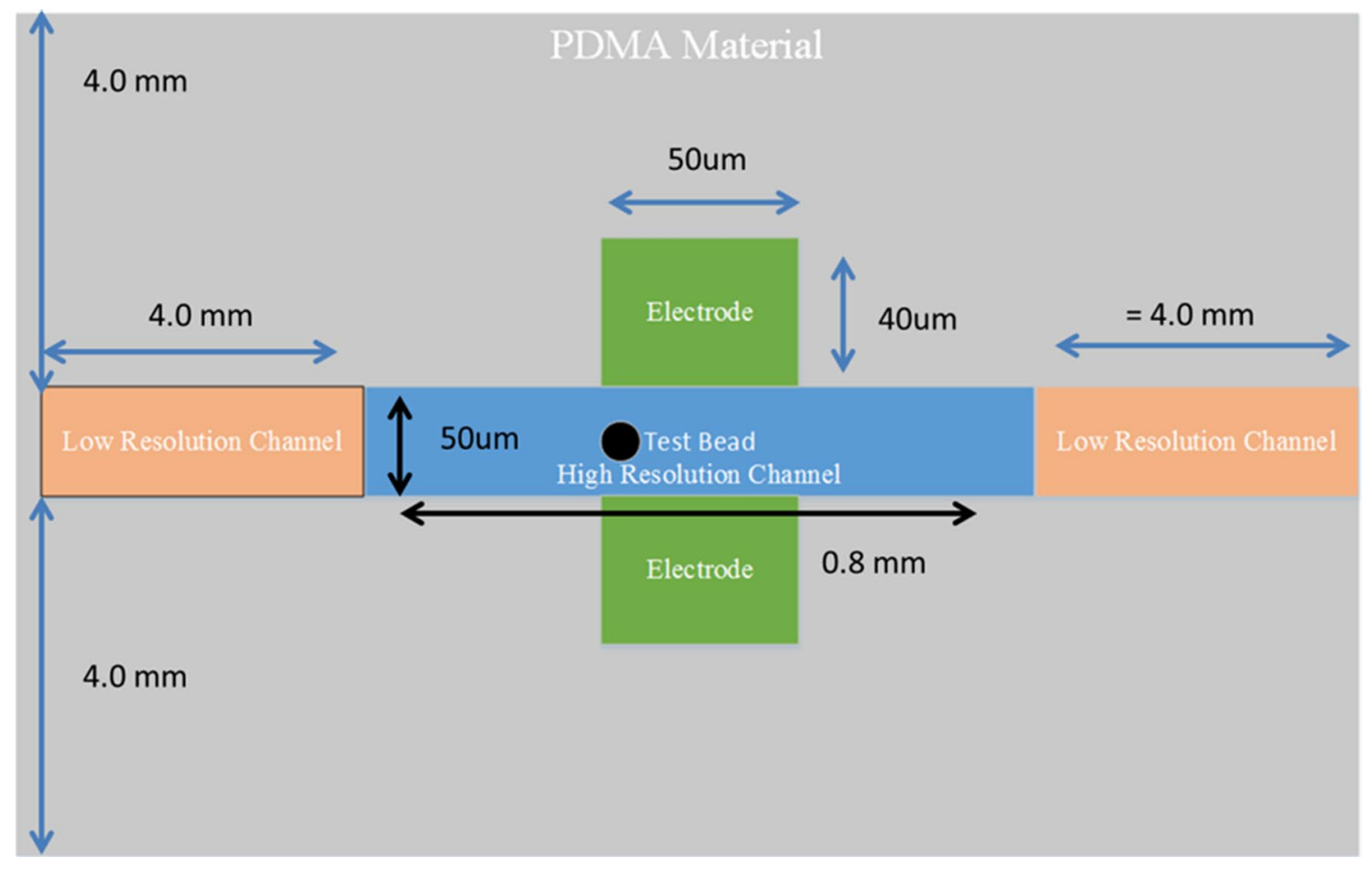

Fig. 10 Dimensions of 2D test model 


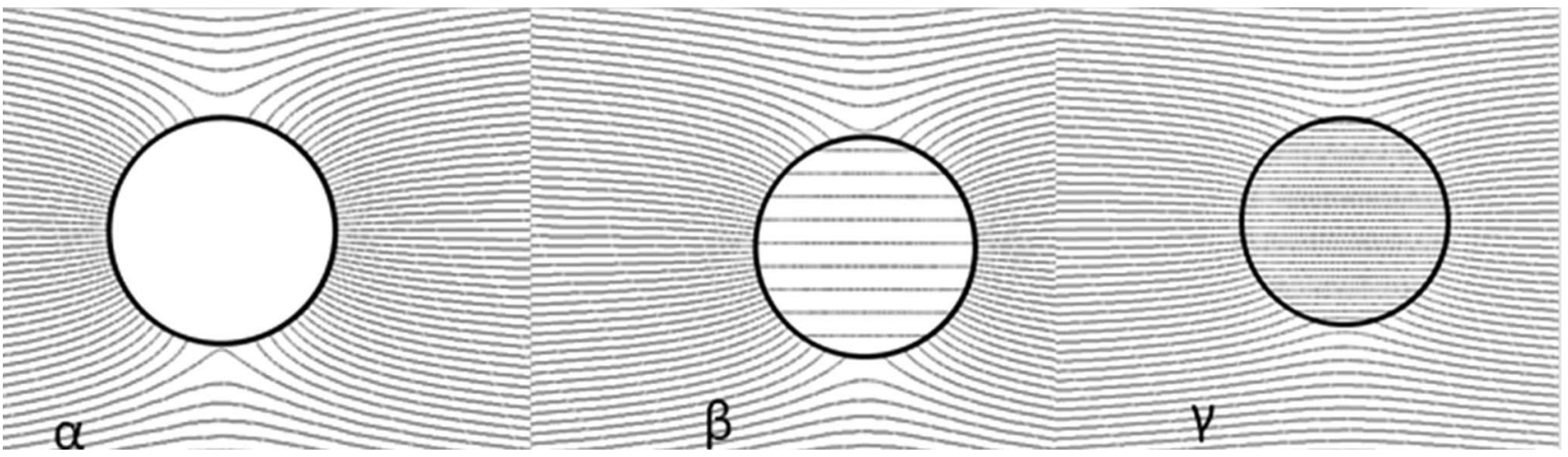

Fig. 11 FEM simulation of bacterium showing frequency dependent responses

- The impact of the membrane for the cell and the double step from the cell wall and membrane for the bacterium model.

- The shift in characteristics as the frequency of the applied field was increased both in terms of the electrode, channel, conductive medium and the cells responses.

The results from a simulated MEMS device based on common aspects of designs used in a number of papers provided an insight into the expected response. Figure 12 shows the resistive losses versus frequency for a central point in a bead, blood cell and bacterium. This highlights the difference between the responses of the homogenous bead and the biological cells.

\subsection{Extracting other information from the FEM}

Other FEM based analyses allowed the evaluation of the overall capacitance of the system (for static cases) and allowed the resonance peaks of the systems to be determined. COMSOL's multiphysics capability allowed the integration of spice models with the physical structures created. COMSOL's MEMS fluidic physics and the AC physics module enabling the temperature increase due to energy absorption to be simulated, allowing power levels and in particular power dissipations into the cells and medium to be examined.

\section{Optimising the MEMS channel structures for sensitivity}

In the non-contactless case, the series capacitances include the EDL effect between the electrodes and the carrier medium. For the contactless case, additional

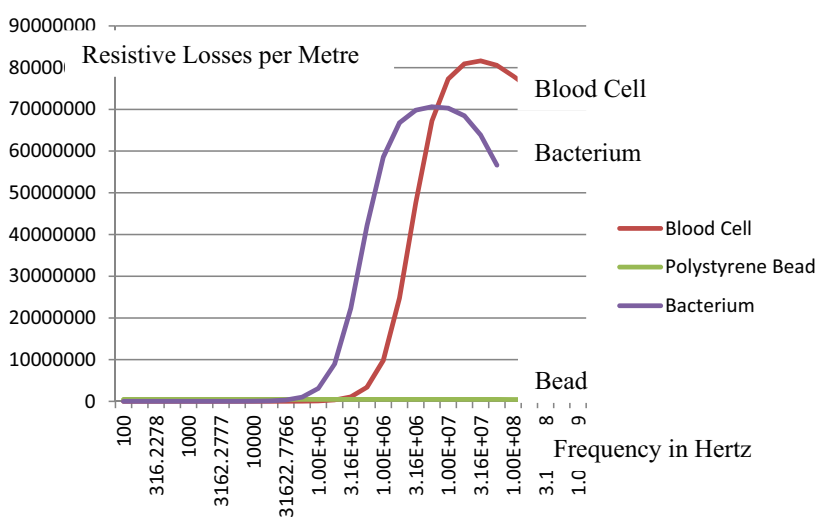

Fig. 12 Simulation results of resistive losses versus frequency for centre of each cell in the 2D test simulation

contributions resulting from the impedance differences between the electrodes, insulating mediums and the channel need to be considered. In terms of design sensitivity, minimisation of the direct inter-electrode coupling and maximisation of the coupling via the channel medium was required. Reducing the direct signal to a minimum allowed the maximum feasible gain on the channel signal to be used. The presence of ground planes and grounded structures in close proximity to the active electrodes complicated the situation significantly. Treating electrodes as isolated parallel plate capacitors produced erroneous figures and in these situations fringing capacitance is a significant contributor. Theoretically to reduce the direct coupling, the maximum separation and minimum overlap between the drive and sense electrodes is required. By combining the cell models considered previously with a basic model for a channel, the electrical system shown in Fig. 13 was derived. The coupling capacitance represents the lumped capacitance of the signal path and stray coupling into the channel. 


\subsection{Optimum positions of electrodes}

In terms of optimum sensitivity, positioning the electrodes so that they are separated by the channel and have no parallel components provides the minimum of direct capacitive coupling. Unfortunately this approach has the highest manufacturing cost, requiring electrodes to be placed in close proximity to, and on both sides of the channel. This is the case shown in Fig. 10. An alternative is to use coplanar electrodes on a single side of the channel. This has the cheapest manufacturing costs, but also the lowest sensitivity and highest direct capacitive coupling between the electrodes. The signal saturation this causes significantly affects the overall sensitivity that can be achieved. Increasing the inter electrode and channel spacing reduces the count rate, channel discrimination and increases the dead time of the system. Poisson derived approaches are commonly used to calculate the optimum channel length and electrode position for carrier concentrations, count rates and dead time.

\subsection{Limitations to maximising the channel impedance}

If we maximise the channel impedance by increasing the length of the channel this has two significant detrimental effects. The first is that this increases the impedance and reduces the signal strength in the channel; the overall percentage impedance change due to a single cell will be significantly reduced. In a narrow channel where the cell obstructs a significant amount of the two dimensional cross section, this is less critical than in a wider channel where the percentage change is significantly less. Secondly, the sensitivity in terms of discrimination between individual cells will decrease. Addressing this requires an increase in separation and hence the overall dilution of the biological material in the carrier medium. A FEM based simulation of the difference between a cross channel and a co-planar approach, for electrodes adjacent to the channel, showed an overall drop of approximately $300 \%$ in the change caused by a red blood cell. The effective separation distance between the electrodes was maintained for these simulations. The cell was located in the centre of the channel equidistant from each electrode. The basic structure of a cross channel MEMS device is shown in Fig. 10 whilst a co-planar one is shown in Fig. 14.

The percentage change in signal strength between the two designs is shown in Fig. 15. Vertical displacement of the cell in the relatively high channel used, $50 \mu \mathrm{m}$, causes significant variation in the signal. Optimisation of the signal chain has to consider a wider range of input values than if the vertical cell placement was constrained to less than the full channel width, or the channel narrowed in some manner.

\subsection{Low cost and disposable MEMS structures}

The majority of approaches investigating low cost disposable MEMS modules, (Sun 2010; Park et al. 2009; Emanminejad et al. 2012) use co-planar approaches separated by a microscope cover slip or places the channel directly on a PCB. The resultant loss in sensitivity requires significant channel narrowing to provide the required signal to noise ratio in these situations. Simulations show that an alternative approach, with a single electrode in the MEMS device and the other separated by a glass cover slip provides significantly increased sensitivity, albeit with an increased manufacturing cost. This may provide an acceptable intermediate approach in reducing the manufacturing cost whilst increasing the sensitivity, allowing more sophisticated analyses to be carried out. An FEM design incorporating a type 0 glass cover slip was developed. Type 0 cover slips have a specified thickness of between 60 and $130 \mu \mathrm{m}$, the worst case thickness

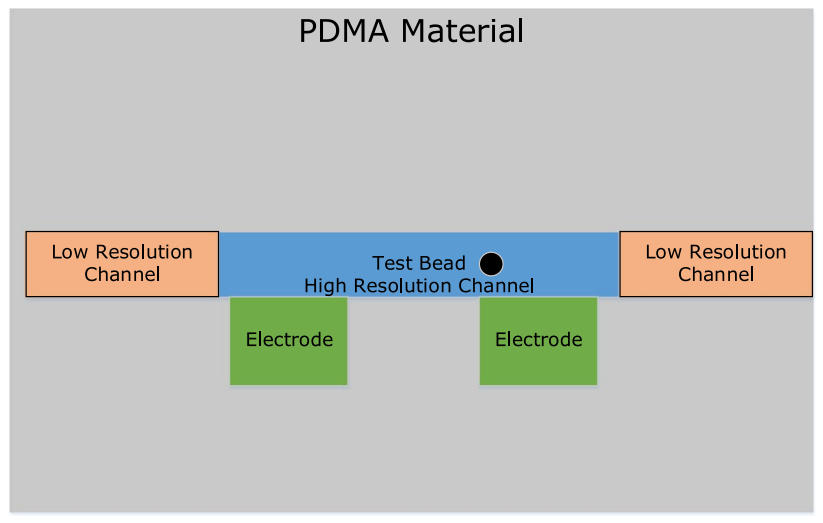

Fig. 14 Co-planar channel MEMS model

Fig. 13 Ten electrical models of channel and cell impedances 
of $130 \mu \mathrm{m}$ was used for the model. Figure 16 shows the basic construction for the co-planar and hybrid designs. Figure 17 shows how this approach results in significant direct coupling between the co-planar electrodes, with minimal energy coupling into the channel.

This causes a high base line current at all frequencies. The overall frequency dependent change in the terminal currents was of the order of $0.14 \%$. The actual detection range where the effect of a cell in the channel could be detected is pushed into the high $\mathrm{MHz}$ regions. The high base line current exacerbating the issues associated with detecting the much reduced change. These characteristics are why dark field resonance and differential approaches dominate the current solutions used in these situations. The difference between the presence of a centrally placed blood cell and a clear channel under the best conditions was in the $10^{-8}$ region for the relative change in terminal current.

\subsection{A hybrid approach}

Empirically a construction where the electric field is across the channel using electrodes on either side will always provide a significantly better response than a coplanar approach. Calculations using inverse square and parallel plate capacitor approaches provide a guide to the performance. The FEM simulations confirm this, providing an enhanced qualitative comparison and making "what if" scenarios easier to explore. A hybrid approach, where one electrode is instantiated in the PDMA as close as possible to the channel and the other is separated by the glass cover slip was considered as an alternative
Fig. 15 Percentage change in detection signal strength for cross channel and co planar arrangements
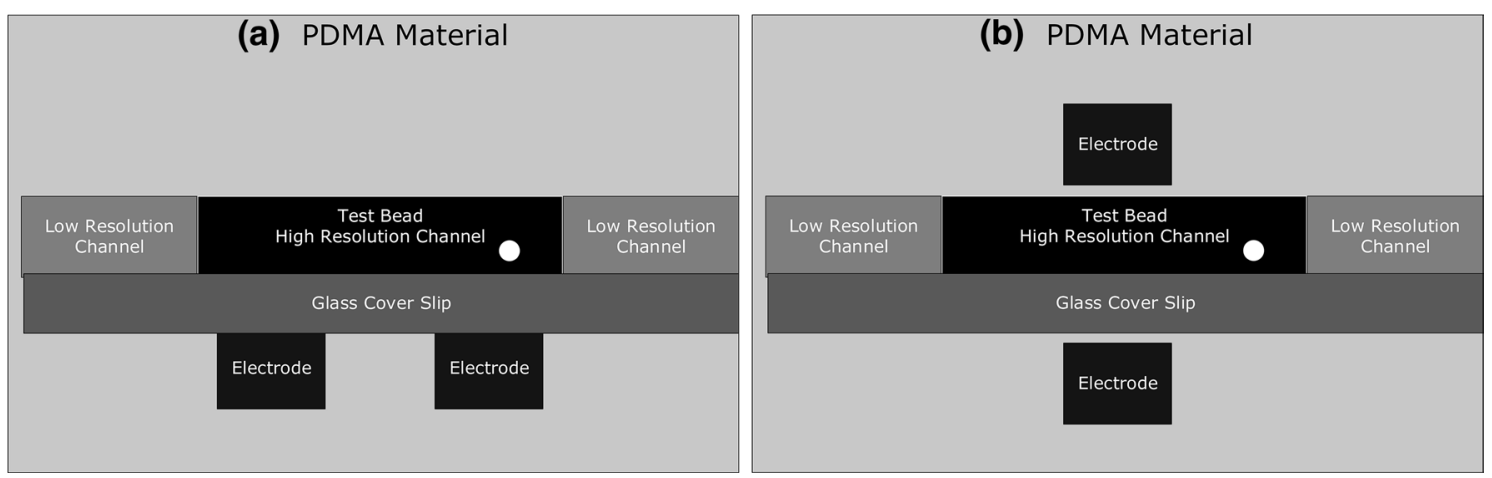

Fig. 16 a, b Co-planar and hybrid low cost modules 
Fig. 17 Terminal current versus frequency for the coplanar design
Fig. 18 Terminal current versus frequency for hybrid cross
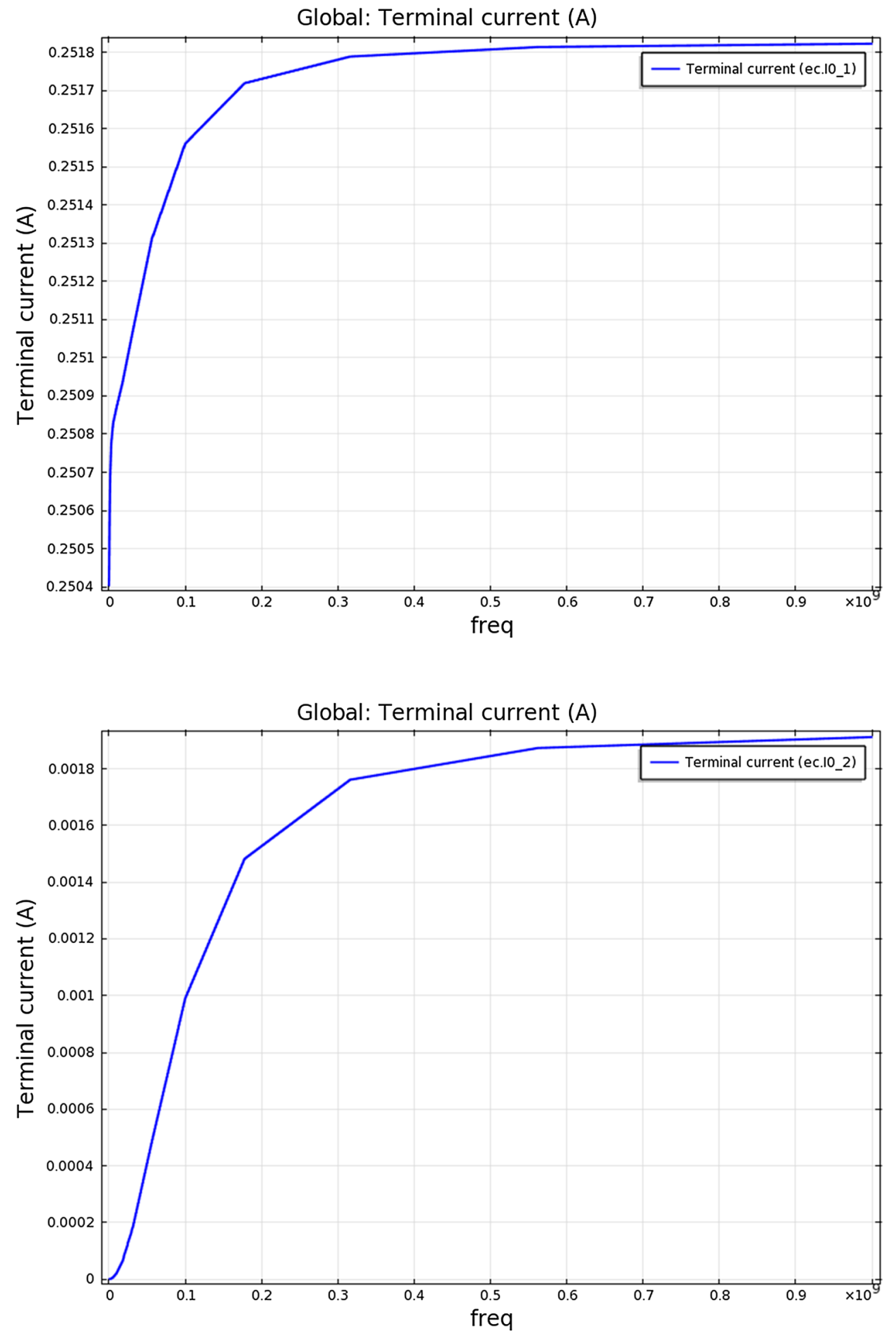

approach. Figure 18 shows the terminal currents in this situation. The electrode in proximity to the channel was used as the drive terminal. The direct coupled inter electrode current magnitude is at least 100 times less than that for the co-planar approach. The percentage change in the terminal current across the frequency range is approximately $900 \%$ providing a dramatically improved detection regime across the range of interest in response to the presence of a red blood cell in the channel.

Table 2 shows the relative terminal current differences between a channel with and without a cell present for the cross and co-planar structures over a range 
Table 2 Frequency dependent difference in terminal current for the cross and hybrid cases

\begin{tabular}{lll}
\hline Frequency hertz & Relative cross hybrid & Relative coplanar \\
\hline $1.0 E+2$ & 0 & 0 \\
$3.16 E+2$ & $-5.6 E-10$ & $4.43 E-14$ \\
$10 E+3$ & $-6.1 E-09$ & $4.43 E-13$ \\
$3.162 E+3$ & $-6.2 E-08$ & $4.21 E-12$ \\
$1.0 E+4$ & $-6.2 E-07$ & $4.44 E-11$ \\
$3.162 E+4$ & $-6.2 E-06$ & $4.21 E-10$ \\
$1.0 E+5$ & $-6.2 E-05$ & $4.43 E-09$ \\
$3.162 E+5$ & -0.00061 & $4.17 E-08$ \\
$1.0 E+6$ & -0.08519 & 0 \\
$3.162 E+6$ & -0.20774 & 0 \\
$1.0 E+7$ & -0.6603 & 0 \\
$3.162 E+7$ & -4.58643 & 0 \\
$1 E+08$ & -28.0575 & 0 \\
$3.16 E+08$ & -68.6985 & 0 \\
$1 E+09$ & -89.6266 & 0.00412 \\
\hline
\end{tabular}

of frequencies. It can be seen from Table 2 that the cross channel responses relative sensitivity was consistently at least 4 orders of magnitude, or $82 \mathrm{~dB}$, above the coplanar response. Nor does it exhibit the "dead zone" that the co-planar simulations did. One would expect any frequency in the low $\mathrm{kHz}$ region to be compromised by $1 / f$ system noise in any case. The lower frequencies require sensitivities that would be difficult or problematic to obtain, limiting low frequency operation. It should be noted that all simulations were done using a uniform $1 \mathrm{~V}$ applied field. The system, certainly at the lower frequencies could deliver up to a $30 \mathrm{~V}$ swing, providing an additional $29 \mathrm{~dB}$ of gain in the signal chain.
The use of nonlinear gain structures would allow tailoring of the signal amplitude across the frequency range. Higher gains could be used at the lower frequencies and reduced for the slew rate limited higher frequencies with increased coupling efficiency. The actual difference in electric fields can be seen in Fig. 19, which highlights the differing intensity of the electric field present between the approaches at $100 \mathrm{MHz}$. The signal path between the drive, cell and sense terminals in the co-planar case being significantly longer. This results in a far weaker field being present in the channel to "detect" the biological elements.

Whilst this approach increases manufacturing complexity, requiring a single electrode to be inserted above the channel. It provides a considerable improvement in sensitivity. Significant variations in the vertical placement of the electrode still results in a response superior to the co-planar case. It is possible to realise a system where a probe could be inserted into a predefined cavity in a low cost MEMS module, or could be physically inserted during manufacture. In a replaceable module, some form of gel to improve coupling might be appropriate to add consistency to the interface, reducing interface variability. The approach appears to retain the desirable features of low complexity, good tolerance to manufacturing variation, robustness and adding little to the cost of module fabrication.

\section{The macroscopic test bench}

In order to allow an evaluation of the system performance, albeit at a macroscopic level, a test bench was created. A commercial TSSOP 14 SMD PCB prototype board was used for the test bench. This gave an inter electrode spacing of $0.25 \mathrm{~mm}$. A $1100 \mu \mathrm{m}$ diameter FEP pipe with a $700 \mu \mathrm{m}$

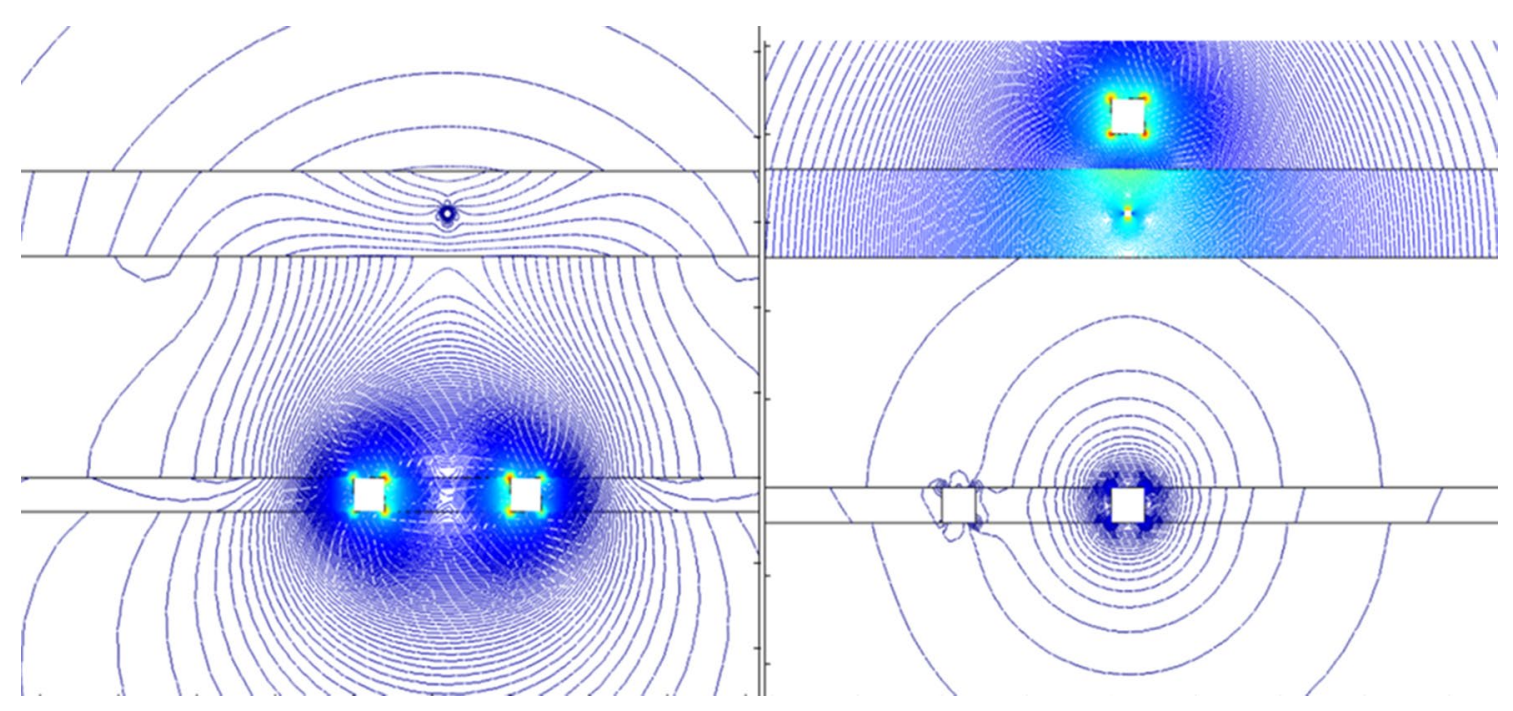

Fig. 19 Field strength comparison between cross and co-planar at $100 \mathrm{MHz}$ for a PBS filled channel 
central channel was mounted cross wise and used as the channel. It was not intended to allow for the reliable detection of particles of the target size to support evaluation of the system. The final structure used was modelled in three dimensions in COMSOL as shown in Fig. 20. This supported evaluation of the performance of the macroscopic system and allowed comparison with a MEMS scaled solution.

\subsection{Electrode spacing on the macroscopic test bench}

As fringing fields and other complex interactions affect the system, an analysis using a FEM approach was carried out. Simulations were run to determine the optimum configuration and frequencies to use. During physical testing, the intention was to fill the tube with either PBS or an insulating mineral oil, creating a significant change in characteristics. The overall results as shown in Table 3 were in line with the results from calculations where a significant gap between the electrodes was required to reduce the direct electrode coupling to acceptable levels. Increasing the separation of the electrodes increased the relative coupling through the channel.

Given the significant increase in sensitivity of the channel impedance, (approximately 600\%), a spacing



Fig. 20 3-dimensional macroscopic test bench FEM

Table 3 Static capacitance results for macroscopic test bench simulation

\begin{tabular}{lll}
\hline Test configuration & Material in tube & Simulated DC capacitance \\
\hline Adjacent pins & Air & $1.3785 e-13$ \\
Adjacent pins & PBS $0.5 \mathrm{Sm}^{-1}$ & $1.3822 e-13$ \\
Spaced by 1 & Air & $1.2703 e-13$ \\
Spaced by 1 & PBS $0.5 \mathrm{Sm}^{-1}$ & $1.2959 e-13$ \\
Spaced by 2 & Air & $1.2213 e-13$ \\
Spaced by 2 & PBS $0.5 \mathrm{Sm}^{-1}$ & $1.2506 e-13$ \\
\hline
\end{tabular}

of 2 electrodes, was used. The "unwanted" terminal was removed from the PCB to reduce noise. Analysis using an AD811 based Gumm oscillator resonance approach gave a capacitance value of the order of $1.5 \times 10^{-9} \mathrm{~F}$, albeit with a not inconsiderable margin of error.

\subsection{Correlation with a MEMS based system}

In the MEMS based system, the structure of the channel monitored has a direct effect on the sensitivity and rate at which cells could be counted. Further simulations and calculations would be needed to determine the optimum configuration for the channel width, height and separation between the electrodes. In particular, the acceptable variation in vertical and horizontal location of the cells will have a significant effect on the lowest viable sensitivity the system can operate with. Simulations were carried out using the two dimensional test structures to allow comparisons with signal strengths, channel sizes and electrode location. These allowed the " $Q$ " of the system to be determined. Allowing the reference frequencies selected to avoid the potential for unwanted interaction with resonance zones. A number of 2 dimensional models were created. These allowed the differences in sensitivity due to the changing location of the electrodes relative to the channel to be analysed across the frequency range. Figure 21 shows the frequency response for the system using the relative response difference between the tube filled with air and PBS. At this scale the difference between PBS and distilled water was too small to allow for discrimination between them.

From Fig. 21 it can be seen that the FEM simulation required a frequency in excess of $5 \mathrm{MHz}$ before sufficient coupling into the channel occured to allow discrimination between the different channel conditions. The results indicate an optimum frequency in the $100 \mathrm{MHz}$ range.

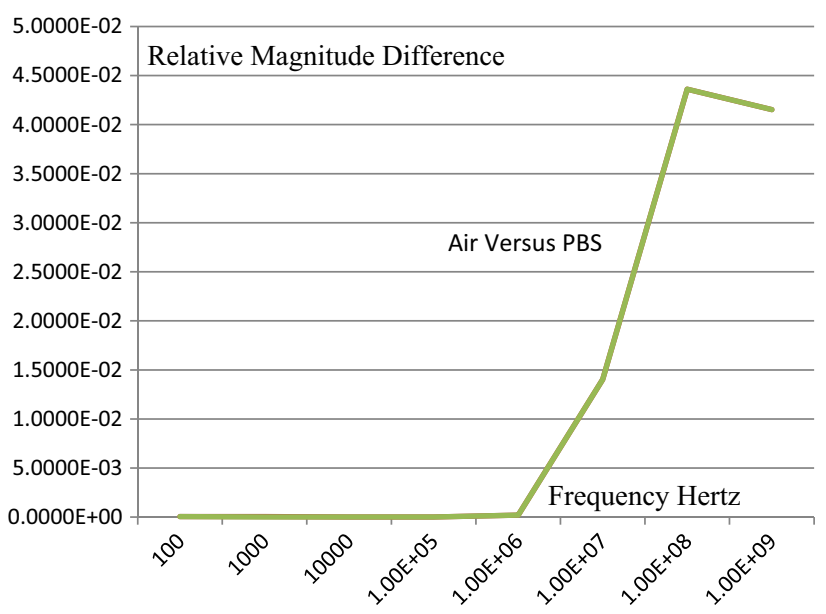

Fig. 21 Relative differences in magnitude for air versus water in channel 
This was borne out in actual testing where even with an $18 \mathrm{~V}$ drive level the response at low frequencies could not be reliably detected. It showed that the difference was significant enough for a self-test function to determine whether the channel contained a liquid or air.

\section{Conclusion}

This paper has presented the successful development of a mixed signal system capable of concurrently generating and measuring $\mathrm{AC}$ characteristics at widely separated multiple frequencies for use in a microfluidic MEMS platform. The approach is energy efficient in that it does not utilise wide band approaches, allowing energy to be channelled into frequencies of interest. It does not require duplication of the analogue signal chains for either the RX or TX subsystems. The requisite VHDL RTL has been developed, simulated and synthesised onto an FPGA platform to support the entire signal chain. The core of the signal chain utilises modern DSP techniques to concurrently process the multiple signals, allowing for significant flexibility. Electrical and electronic subsystems such as A2D, D2A, control logic and LCD blocks were developed and integrated into the working design. An RTL design approach is described that allowed the maximum frequency range to be significantly increased without requiring a corresponding increase in the system clock rate. This facility provided an increased signal range and improved sensitivity in the digital phase quadrature blocks of the signal chain. The entire signal chain has been demonstrated as a working system.

A fully functioning test bench using macroscopic structures has been used to demonstrate the viability of the approach, allowing identification of air/pbs/mineral oil in the channel. In parallel with this, FEM models were developed to investigate the performance of the proposed techniques as well as allowing comparison between the existing macro scale and proposed microfluidic MEMS platforms.

Analysis of the performance and structure of existing microfluidic MEMS platform solutions resulted in a proposal for a new structure. FEM analysis showed that the proposed hybrid, cross channel electrode structure, provided significantly enhanced sensitivity, whilst potentially having minimal impact on manufacturing complexity and cost.

The synthesisable portion of the design was successfully implemented within the relatively modest resources, both in terms of gates and clock rate, of a cyclone IIbased Altera DE1 development board.

\section{Future developments}

A number of approaches to increase the frequency range, discrimination and resolution of the system have been discussed including mixed signal and DSP based techniques.

Approaches allowing the use of higher resolution converters, either by using an up down conversion or subNyquist sampling approaches should be considered to allow improvements in the signal to noise ratio of the signal chain (Sun et al. 2009).

The use of advanced DSP filters such as Gaussian, adaptive or moving average should provide improved discrimination in comparison to the current low pass filter approach.

\section{Compliance with ethical standards}

Conflict of interest The authors declare they have no conflict of interest.

Open Access This article is distributed under the terms of the Creative Commons Attribution 4.0 International License (http://creativecommons.org/licenses/by/4.0/), which permits unrestricted use, distribution, and reproduction in any medium, provided you give appropriate credit to the original author(s) and the source, provide a link to the Creative Commons license, and indicate if changes were made.

\section{References}

Andraka R (1998) A survey of CORDIC algorithms for FPGA based computers. In: FPGA 98, pp 191-200

Beving H, Eriksson L, Davey C, Kell D (1994) Dielectric properties of human blood and erthroycytes at radio frequencies $(0.210$ $\mathrm{MHz}$ ); dependence on cell volume fraction and medium composition. Eur J Biophys 23:207-215

Britt WB, Hoffman RA (1979) Flow system measurement of cell impedance properties. J Histochem Cytochem 27(1):234-240

Chen CC, Lin PH, Chung CK (2014) Microfluidic chip for plasma separation from undiluted human whole blood samples using low voltage contactless dielectrophoresis and capillary force. Lab Chip 14(12):1996-2001. doi:10.1039/C4LC00196F

Cole R, Cole K (1941) Dispersion and absorption in dielectrics I alternating current characteristics. J Chem Phys 9:341-351

Coulter W (1956) High speed automatic blood cell counter and cell size analyzer. In: The national electronics conference, October 3rd 1953, Chicago, USA

Debye P (1945) Polar molecules. Dover, New York (Online)

Emanminejad S, Javanmard M, Dutton R, Davis R (2012) Microfluidic diaganostic tool for the developing world: contact less impedance flow cytometry. Lab Chip 12:4499-4507

Fricke H (1953) The Maxwell Wagner dispersion in an suspension of ellipsoids. J Phys Chem 57(9):934-937

Gimsa DWJ (1998) A unified resistor-capacitor model for impedance, dielectropheresis, electrotation and induced transmembrane potential. Biophys J 75(1):1107-1116

Holmes D, Gawad S, Green NG, Sun HMT (2007) High speed multi frequency impedance analysis of single particles in a microfluidic cytometer using maximum length sequences. Lab Chip 7:1034-1040 
Hu YH (1992) CORDIC based VLSI architectures for digital signal processing. IEEE Signal Process Mag 9(3):16-35. doi:10.1109/79.143467

Javanmard M, Dutton RW, Davis RW, Emaminejad S (2012) Microfluidic diagnostic tool for the developing world: contactless impedance flow cytometry. Lab Chip 12:4499-4507

Johnson TS, Britt WB, Hoffman RA (1980) Flow cytometric electronic direct current volume and radio frequency measurement of single cell particles. Cytometry 1(6):377-384

Min M, Paavle T, Annus P, Land R (2009) Rectangular wave excitation in wideband bioimpedance spectroscopy. In: MeMea 2009, Cetrano, Italy, pp 268-270

Neeman S (2004) The frequency distribution of quantisation error in digitizers for coherent sampling. In: Proceedings of the second (IASTED) International conference on circuits, signals, and systems, Nov 28-Dec 1 2004. Clearwater Beach, FL, USA, pp 35-39

Nieuwstadt HA, Seda R, Li DS, et al (2011) Microfluidic particle sorting utilizing inertial lift force. Biomed Microdevices 13:97. doi:10.1007/s10544-010-9474-6

Park K, Suk HJ, Akin D, Bashir R (2009) Dielectophoresis-based cell manipulation on a reusuable printed circuit board. Lab Chip 9:2224-2225

Park S, Zhang Y, Wang TH, Yang Samuel (2011) Continous dielectrophoretic bacterial separation and concentration from physiological media of high conductivity. R Soc Chem Lab Chip 11(17):2893-2900

Ristovic M, Lubura S, Jokie D (2012) Implementation of CORDIC algorithm on FPGA Altera cyclone. In: Telefor, Belgrade, pp 875-878
Spencer D, Benazzi G, Mowlem MC, Barat HMD (2012) Simultaneous high speed optical and impedance analysis of single particles with a microfluidic cytometer. Lab Chip 12:118-126

Sun HMT (2010) Single cell microfluidic impedance cytometry a review. Microfluide Nanafluid 8:423-443. doi:10.1007/ s10404-010-0580-9

Sun T, Holmes D, Gawad S, Green NG, Morgan H (2007) High speed multi frequency impedance analysis of single particles in a microfluidic cytometer using maximum length sequences. Lab Chip 7:1034-1040

Sun T, Berkel C, Green N, Morgan H (2009) Digital signal processing methods for impedance microfluidic cytometry. Microfluid Nanofluid 6:179-187

Ulgen Y, Sezdi M (1998) Electrical parameters of human blood. In: 20th annual conference of the IEEE Engineering in Medicine and Biology Society, vol 20, pp 2983-2986

Upadhyay R, Sarawade N, Varde S (2013) Simulink design of pipelined CORDIC for generation of sine and cosine values. Int J Comput Eng Res 3(3):312-316

Van Berkel C, Green NG, Sun HMT (2009) Digital signal processing methods for impedance microfluidic cytometery. Microfluid Nanofluid 6(2):179-187

Volder J (1959) The CORDIC trigonometric computing technique. IRE Trans Electron Comput EC-8:330-334

Wolf M, Gulich R, Lunkenheimer P, Loidl A (2011) Broadband dielectric spectroscopy on human blood. Biochimica et Biophysics Acta (BBA) Gen Subj 1810(8):727-740

Wood DK, Oh SH, Soh HT, Cleland AN (2005) High-bandwidth radio frequency Coulter counter. Appl Phys Lett 87:184106 